Marcus J. Schultz Peter E. Spronk

\section{Should mechanical ventilation care be centralized and should we thus transfer all ventilated patients to high volume units? Take a breath first}

Received: 6 January 2014

Accepted: 11 January 2014

Published online: 7 February 2014

(C) Springer-Verlag Berlin Heidelberg and ESICM 2014

M. J. Schultz (®) · P. E. Spronk

Department of Intensive Care C3-415, Academic Medical Center, University of Amsterdam, Meibergdreef 9, 1105 AZ Amsterdam, The Netherlands

e-mail: marcus.j.schultz@gmail.com

M. J. Schultz

Laboratory of Experimental Intensive Care and Anesthesiology, Academic Medical Center, University of Amsterdam, Amsterdam, The Netherlands

P. E. Spronk

Department of Intensive Care, Gelre Hospitals, Apeldoorn, The Netherlands

For years, many critical care physicians considered mechanical ventilation as an easy and harmless intervention in intensive care unit (ICU) patients. Nowadays, preclinical and clinical researchers and critical care physicians increasingly appreciate the potential harmful effects of ventilation, in both patients with the acute respiratory distress syndrome (ARDS) [1] and those with uninjured lungs [2]. Ventilation turns out to be everything but safe, as it can cause so-called 'ventilator-induced lung injury' (VILI) [3] and 'ventilator-induced diaphragm dysfunction' (VIDD) [4]. Mitigating this harm is far from simple. It requires skill, which like all skills improves with experience.

One steady observation in ICU medicine is the finding that ICUs that care for high volumes of patients experience improved outcomes. This finding, also known as the 'volume-outcome relationship,' has been demonstrated in several cohorts of patients [5], including those who are ventilated [6]. However, the latter finding is far from settled since half of the studies on this matter could not confirm this relationship [7-11]. In an attempt to further evaluate the potential effect of ICU volume on the outcomes of ventilated admissions, Shahin et al. [12] retrospectively evaluated the volume-outcome relationship for ventilated patients to adult, general ICUs. They extracted data for the years 2008-2010 from the case mix program database (CMPD), a registry that contains pooled case mix and outcome data on consecutive admissions to ICUs in England, Wales, and Northern Ireland. The primary exposure of interest was annual volume of ventilated admissions per ICU per year; the primary outcome was ultimate acute hospital mortality.

After adjusting for potential confounders including, but not restricted to, age, gender, disease severity, and comorbidities, Shahin et al. found a significant relationship between annual volume and ultimate acute hospital mortality, with a higher volume of ventilated admissions associated with lower mortality. Interestingly, a stronger interaction was found for non-surgical patients, but the relationship was not affected by the severity of respiratory failure.

Do these results suggest that we should centralize mechanical ventilation care in the hope to improve ICU outcome? This suggestion, in itself an obvious one, may be misleading for several reasons. First of all, Shahin et al. report on the relationship between ICU volume and hospital outcome, while the relationship with ICU outcome is not reported. If the found volume-outcome relationship depends on differences in ventilation practice, as suggested by the authors themselves, a relationship between ICU volume and ICU length of stay and maybe even ICU mortality would exist as well $[1,2]$. Furthermore, the analysis did not correct for important 
potential confounders, such as the availability of around the clock critical care physicians, the intensity of staffing, the presence of multidisciplinary care teams, the use of sedation, blood transfusion and fluid resuscitation protocols, and availability of intervention radiologists and cardiologists, to name a few (Fig. 1) [13, 14]. All of these have a strong potential to affect outcomes, and differences between centers, depending on size, can be presumed. Indeed, one major unknown in the context of many volume-outcome relationships is the underlying mechanism [15]. Future studies should address this issue, although this may be very hard if not impossible.

Second, one important challenge with studies using registries is that 'registries register what they register' and as such could miss important information. The CMPD collects important information such as admission and physical data during the first $24 \mathrm{~h}$ of admission and ICU and hospital outcome data, but fails to capture data during stay in the ICU and hospital beyond $24 \mathrm{~h} \mathrm{[16].} \mathrm{For}$ instance, the clinical presentation of VILI may take some time, appearing after the first day of ventilation $[17,18]$. Could this be the reason why Shahin et al. did not find a stronger relationship in patients with more severe respiratory failure? Again, if the found volume-outcome relationship depends on differences in ventilation practice, a larger effect would have been expected in these high-risk patients. This is not found, though.

Let us assume that an independent effect of case volume on the final outcome does exist; this bears the question: what would happen if we transfer ventilated patients to a larger center-does this actually improve outcome? Understandingly, to avoid confounding results with outcomes from other units, Shahin et al. excluded the admissions transferred either directly into a unit from another critical care unit or those transferred out (within $24 \mathrm{~h}$ ) directly to another critical care unit. As the authors themselves suggest, transference of ICU could have important negative effects as well. A case-control study of those patients would have provided us with an answer to this important question, though. And which patients would benefit most from such transfers-patients who need ventilation for more than $24 \mathrm{~h}, 48 \mathrm{~h}$ or 1 week? We do not know the answer to these important questions.

Two essential things should not be forgotten in the discussion of centralization of ventilation care. First, admissions to the ICU are almost always unplanned, and harm by ventilation can already be achieved within a short period, that is: within hours [2]. This means that if we really wanted to centralize mechanical ventilation care, many patients would remain at risk for harm by ventilation, i.e., before ICU transfer-unless we close those hospitals with low volume ICUs, which will probably never happen. Second, a lower ICU occupancy rate with inherent lower exposure to ventilated patients, such as in many areas of the USA or Australia $[19,20]$, bears the risk of losing experience with safe ventilation with a concomitant increased risk of unwanted side effects.

If the rule 'practice makes perfect' indeed applies for ventilation, what else can we do to improve the outcomes of ventilated ICU patients? A more practical solution
Fig. 1 Schematic view of factors that potentially affect the 'volume-outcome relationship' for ventilated ICU patients. Most studies of the 'volume-outcome relationship' restricted confounders to those like case mix and admission volume, while numerous factors before, during and after stay in the ICU can have a substantial effect on ICU or hospital mortality. This schematic largely focuses on factors associated with (reasons for) mechanical ventilation. $M V$ mechanical ventilation, $E C \mathrm{CO}_{2} \mathrm{R}$ extracorporal $\mathrm{CO}_{2}$ removal, ECMO extracorporal membrane oxygenation, VAP ventilator-associated pneumonia, CAP communityacquired pneumonia, HAP hospital-acquired pneumonia

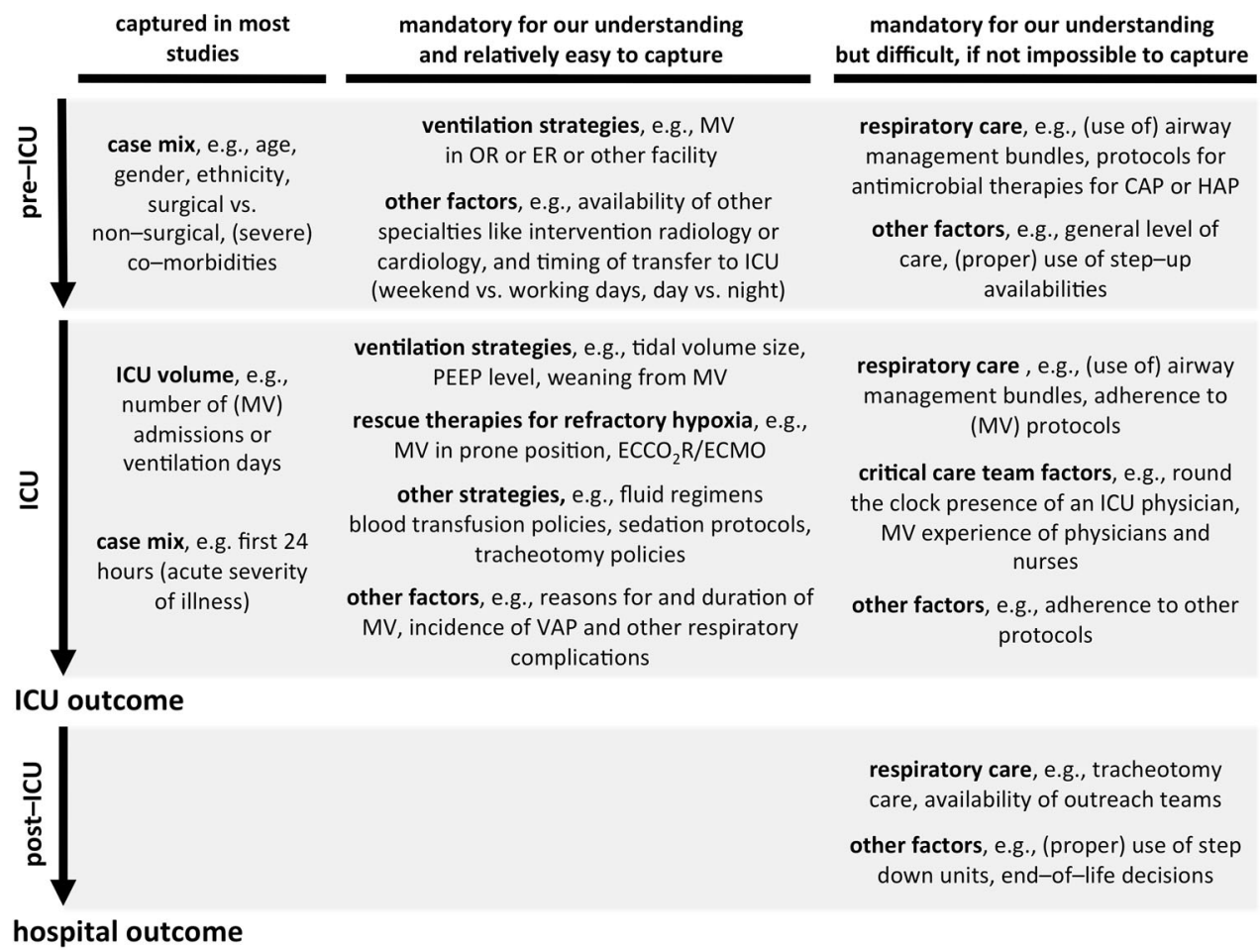


might simply be to intensify education on safe ventilation and to implement practicable protocols of protective ventilation. And last but not least, all critical care team members, i.e., critical care physicians, critical care nurses, and respiratory therapists, must understand that ventilation is a potentially harmful intervention, regardless of admission type and duration of ventilation.

\section{References}

1. Putensen C, Theuerkauf N, Zinserling J, Wrigge H, Pelosi P (2009) Metaanalysis: ventilation strategies and outcomes of the acute respiratory distress syndrome and acute lung injury. Ann Int Med 151:566-576

2. Serpa Neto A, Cardoso SO, Manetta JA, Pereira VG, Esposito DC, Pasqualucci Mde O, Damasceno MC, Schultz MJ (2012) Association between use of lung-protective ventilation with lower tidal volumes and clinical outcomes among patients without acute respiratory distress syndrome: a metaanalysis. JAMA 308:1651-1659

3. Slutsky AS, Ranieri VM (2013) Ventilator-induced lung injury. New Engl J Med 369:2126-2136

4. Levine S, Nguyen T, Taylor N, Friscia ME, Budak MT, Rothenberg P, Zhu J, Sachdeva R, Sonnad S, Kaiser LR, Rubinstein NA, Powers SK, Shrager JB (2008) Rapid disuse atrophy of diaphragm fibers in mechanically ventilated humans. New Engl J Med 358:1327-1335

5. Kanhere MH, Kanhere HA, Cameron A, Maddern GJ (2012) Does patient volume affect clinical outcomes in adult intensive care units? Intensive Care Med 38:741-751

6. Kahn JM, Goss CH, Heagerty PJ, Kramer AA, O'Brien CR, Rubenfeld GD (2006) Hospital volume and the outcomes of mechanical ventilation. New Engl J Med 355:41-50

7. Halm EA, Lee C, Chassin MR (2002) Is volume related to outcome in health care? A systematic review and methodologic critique of the literature. Ann Int Med 137:511-520

8. Gopal S, O'Brien R, Pooni J (2011) The relationship between hospital volume and mortality following mechanical ventilation in the intensive care unit. Minerva Anestesiol 77:26-32
9. Needham DM, Bronskill SE, Rothwell DM, Sibbald WJ, Pronovost PJ, Laupacis A, Stukel TA (2006) Hospital volume and mortality for mechanical ventilation of medical and surgical patients: a population-based analysis using administrative data. Crit Care Med 34:2349-2354

10. Moran JL, Solomon PJ, Outcome ACf, Resource evaluation of the A, New Zealand Intensive Care S (2012) Mortality and intensive care volume in ventilated patients from 1995 to 2009 in the Australian and New Zealand binational adult patient intensive care database*. Crit Care Med 40:800-812

11. Fernandez R, Altaba S, Cabre L, Lacueva V, Santos A, Solsona JF, Anon JM, Catalan RM, Gutierrez MJ, Fernandez-Cid R, Gomez-Tello V, Curiel E, Fernandez-Mondejar E, Oliva JC, Tizon AI, Gonzalez J, Monedero P, Sanchez MG, de la Torre MV, Ibanez P, Frutos F, Del Nogal F, Gomez MJ, Marcos A, Vera P, Serrano JM, Umaran I, Carrillo A, Lopez-Pueyo MJ, Rascado P, Balerdi B, Suberviola B, Hernandez G, Sabadell Score G (2013) Relationship between volume and survival in closed intensive care units is weak and apparent only in mechanically ventilated patients. Anesthesiology 119:871-879

12. Shahin J, Harrison DA, Rowan KM (2014) Is the volume of mechanically ventilated admissions to UK critical care units associated with improved outcomes? Intensive Care Med. doi: 10.1007/s00134-013-3205-4

13. Antonelli M, Bonten M, Chastre J, Citerio G, Conti G, Curtis JR, De Backer D, Hedenstierna G, Joannidis M, Macrae D, Mancebo J, Maggiore SM, Mebazaa A, Preiser JC, Rocco P, Timsit JF, Wernerman J, Zhang H (2012) Year in review in intensive care medicine 2011. II. Cardiovascular, infections, pneumonia and sepsis, critical care organization and outcome, education, ultrasonography, metabolism and coagulation. Intensive Care Med $38: 345-358$
14. Antonelli M, Bonten M, Chastre J, Citerio G, Conti G, Curtis JR, De Backer D, Hedenstierna G, Joannidis M, Macrae D, Mancebo J, Maggiore SM, Mebazaa A, Preiser JC, Rocco P, Timsit JF, Wernerman J, Zhang $\mathrm{H}$ (2012) Year in review in intensive care medicine 2011: III. ARDS and ECMO, weaning, mechanical ventilation, noninvasive ventilation, pediatrics and miscellanea. Intensive Care Med 38:542-556

15. Kahn JM (2013) What's new in ICU volume-outcome relationships? Intensive Care Med 39:1635-1637

16. Harrison DA, Brady AR, Rowan K (2004) Case mix, outcome and length of stay for admissions to adult, general critical care units in England, Wales and Northern Ireland: the intensive care national audit \& research centre case mix programme database. Crit Care 8:R99-R111

17. Determann RM, Royakkers A, Wolthuis EK, Vlaar AP, Choi G, Paulus F, Hofstra JJ, de Graaff MJ, Korevaar JC, Schultz MJ (2010) Ventilation with lower tidal volumes as compared with conventional tidal volumes for patients without acute lung injury: a preventive randomized controlled trial. Crit Care 14:R1

18. Gajic O, Dara SI, Mendez JL, Adesanya AO, Festic E, Caples SM, Rana R, St Sauver JL, Lymp JF, Afessa B, Hubmayr RD (2004) Ventilatorassociated lung injury in patients without acute lung injury at the onset of mechanical ventilation. Crit Care Med 32:1817-1824

19. Wunsch H, Wagner J, Herlim M, Chong DH, Kramer AA, Halpern SD (2013) ICU occupancy and mechanical ventilator use in the United States*. Crit Care Med 41:2712-2719

20. Tierney LT, Conroy KM (2013) Optimal occupancy in the ICU: a literature review. Aust Crit Care Off J Confed Aust Crit Care Nurses 